\title{
The implementation of the boundary element method to the Helmholtz equation of acoustics
}

\author{
S. A. Sivaka, Post-Graduate Student, orcid.org/0000-0003-4740-2210, siwakserg@yandex.ru \\ M. E. Royaka, Dr. Sc., Tech., Professor, orcid.org/0000-0001-8304-7784 \\ I. M. Stupakova, PhD, Tech., Associate Professor, orcid.org/0000-0003-1094-3961 \\ E. S. Voznuka, Post-Graduate Student, orcid.org/0000-0001-7362-6002 \\ A. S. Aleksashina, Master Student, orcid.org/0000-0003-1871-5517 \\ aNovosibirsk State Technical University, 20, K. Marksa St., 630073, Novosibirsk, Russian Federation
}

\begin{abstract}
Introduction: To solve the Helmholtz equation is important for the branches of engineering that require the simulation of wave phenomenon. Numerical methods allow effectiveness' enhancing of the related computations. Methods: To find a numerical solution of the Helmholtz equation one may apply the boundary element method. Only the surface mesh constructed for the boundary of the three-dimensional domain of interest must be supplied to make the computations possible. This method's trait makes it possible to conduct numerical experiments in the regions which are external in relation to some Euclidian three-dimensional subdomain bounded in the three-dimensional space. The later also provides the opportunity of not using additional geometric techniques to consider the infinitely distant boundary. However, it's only possible to use the boundary element methods either for the homogeneous domains or for the domains composed out of adjacent homogeneous subdomains. Results: The implementation of the boundary element method was committed in the program complex named Quasar. The discrepancy between the analytic solution approximation and the numerical results computed through the boundary element method for internal and external boundary value problems was analyzed. The results computed via the finite element method for the model boundary value problems are also provided for the purpose of the comparative analysis done between these two approaches. Practical relevance: The method gives an opportunity to solve the Helmholtz equation in an unbounded region which is a significant advantage over the numerical methods requiring the volume discretization of computational domains in general and over the finite element method in particular. Discussion: It is planned to make a coupling of the two methods for the purpose of providing the opportunity to conduct the computations in the complex regions with unbounded homogeneous subdomain and subdomains with substantial inhomogeneity inside.
\end{abstract}

Keywords - boundary element method, finite element method, the Helmholtz equation, acoustics.

For citation: Sivak S. A., Royak M. E., Stupakov I. M., Voznuk E. S., Aleksashin A. S. The implementation of the boundary element method to the Helmholtz equation of acoustics. Informatsionno-upravliaiushchie sistemy [Information and Control Systems], 2021, no. 2, pp. 13-19. doi:10.31799/1684-8853-2021-2-13-19

\section{Introduction}

One of the most vastly used instruments applied to solve differential equations is the boundary element method (BEM) because it's possible to use BEM for the computations in regions with infinitely distant boundaries in order to find a solution to the so-called external boundary problem. This is particularly important for the problems of wave propagation.

The method has been in use for a long time. Its first mentions may be found in [1-5]. These papers consider the collocation version of BEM. In present days, the versions of BEM based on Galerkin's form are much more preferred. Probably, the first papers describing these versions are [6-9].

There're two versions of BEM: direct and indirect. The direct method is based on the so-called reciprocity relation, which may be seen while analyzing problems based on the concept of fundamental solution of differential equation. For example, the classic work describing BEM in the mentioned context is [10]. The indirect approach of BEM applied to the problem of acoustics is described, for instance, in [11]. In our work, the direct version of $\mathrm{BEM}$ is under consideration.
The classic disadvantage of the original versions of BEM is the necessity to work with dense matrices of SLAE produced by the method. Hence there are many different techniques helping to minimize the asymptotic complexity of BEM while working with such matrices. The techniques are the method of T-spline curves [12], the wavelet approach [13-16], the adaptive cross-approximation $[10,17-19]$ and the fast multipole method [2024].

It is also worth mentioning that BEM is only capable of handling the cases of the domains that can be decomposed into subdomains with homogeneous media. However, it's possible to get rid of this problem by coupling BEM with FEM (finite element method) so that significantly inhomogeneous domains are handled by the FEM part and the remaining domains are taken care of by BEM. Such coupling is done in [25-29].

\section{The Helmholtz equation of acoustics}

The wave equation in a homogeneous medium $\Omega$ is of the form: 


$$
\Delta u(\mathbf{x}, t)=\frac{\partial^{2} u(\mathbf{x}, t)}{v^{2} \partial t^{2}}+F(\mathbf{x}, t), \mathbf{x} \in \Omega .
$$

The parameters of (1) in terms of acoustics can be seen as follows: $u$ - velocity potential; $\mathbf{x}-$ point-vector in space; $t$ - time; $v$ - speed of sound in the medium; $F$ - intensity-function of volume sources of sound; $\Omega$ - the homogeneous domain where the problem (1) is to be solved [30].

For the boundary $\Gamma$ of the domain $\Omega$ let the following be true:

$$
\Gamma=\Gamma_{1} \cup \Gamma_{2}, \Gamma_{1} \cap \Gamma_{2}=\varnothing .
$$

The boundary conditions on $\Gamma_{1}$ and $\Gamma_{2}$ are written as follows:

$$
\begin{gathered}
\left.u(\mathbf{x}, t)\right|_{\mathbf{x} \in \Gamma_{1}}=F_{D}(\mathbf{x}, t) \\
\left.\frac{\partial u(\mathbf{x}, t)}{\partial \mathbf{n}}\right|_{\mathbf{x} \in \Gamma_{2}}=F_{N}(\mathbf{x}, t),
\end{gathered}
$$

where $\mathbf{n}$ is a normal vector defined on $\Gamma$ and external with respect to $\Omega$.

Suppose for the functions in (1)-(3) the following representation is justified:

$$
\begin{gathered}
F(\mathbf{x}, t)=F(\mathbf{x}) e^{i \omega t} \\
F_{D}(\mathbf{x}, t)=F_{D}(\mathbf{x}) e^{i \omega t} \\
F_{N}(\mathbf{x}, t)=F_{N}(\mathbf{x}) e^{i \omega t},
\end{gathered}
$$

where $i$ designates the imaginary unit; $\omega$ is the angular frequency.

As a corollary of (4)-(6):

$$
u(\mathbf{x}, t)=u(\mathbf{x}) e^{i \omega t} .
$$

Substituting (7) into (1) one derives the Helmholtz equation:

$$
\Delta u(\mathbf{x})+k^{2} u(\mathbf{x})=F(\mathbf{x}), k=\frac{\omega}{v} .
$$

The boundary conditions then may be represented accordingly:

$$
\begin{gathered}
\left.u(\mathbf{x})\right|_{\mathbf{x} \in \Gamma_{1}}=F_{D}(\mathbf{x}) \\
\left.\frac{\partial u(\mathbf{x})}{\partial \mathbf{n}}\right|_{\mathbf{x} \in \Gamma_{2}}=F_{N}(\mathbf{x}) .
\end{gathered}
$$

For what follows next, suppose that $F=0$, so there's no volume sources of sound waves in $\Omega$.

\section{The boundary element method}

The method exploits the boundary representation of the unknown function $u$ (8) implementing the concept of the so-called trace operators. Let us define the trace operators for the domain $\Omega$ : the Dirichlet trace $\gamma_{0}^{\Omega}$ and the Neumann trace $\gamma_{1}^{\Omega}$ :

$$
\begin{gathered}
\left(\gamma_{0}^{\Omega} u\right)(\mathbf{x})=\lim _{\mathbf{r} \in \Omega, \mathbf{r} \rightarrow \mathbf{x}} u(\mathbf{r}), \mathbf{x} \in \Gamma ; \\
\left(\gamma_{1}^{\Omega} u\right)(\mathbf{x})=\lim _{\mathbf{r} \in \Omega, \mathbf{r} \rightarrow \mathbf{x}} \mathbf{n}(\mathbf{x}) \cdot \nabla u(\mathbf{r}), \mathbf{x} \in \Gamma,
\end{gathered}
$$

where $\mathbf{n}$ is the unit normal vector specified for the point $\mathbf{x}$ on $\Gamma$ and it's directed to the outside of $\Omega$.

The resulting function of the Dirichlet trace operator applied to the function $u$ is called the Dirichlet data and is designated as $\gamma_{0}^{\Omega} u$, whereas $\gamma_{1}^{\Omega} u$ stands for the Neumann data respectively.

"The solution $u$ to the equation (8) inside $\Omega$ can be expressed by using Green's theorem and the trace operators defined in (11)-(12) [10]"

$$
\begin{gathered}
u(\mathbf{y})=\int_{\mathbf{x} \in \Gamma} G_{k}(\mathbf{y}, \mathbf{x}) \gamma_{1}^{\Omega} u(\mathbf{x}) \mathrm{d} s_{\mathbf{x}}- \\
-\int_{\mathbf{x} \in \Gamma} \gamma_{1, \mathbf{x}}^{\Omega} G_{k}(\mathbf{y}, \mathbf{x}) \gamma_{0}^{\Omega} u(\mathbf{x}) \mathrm{d} s_{\mathbf{x}},
\end{gathered}
$$

where $G_{k}$ is the fundamental solution of the Helmholtz equation:

$$
G_{k}(\mathbf{y}, \mathbf{x})=\frac{e^{i k\|\mathbf{x}-\mathbf{y}\|}}{4 \pi\|\mathbf{x}-\mathbf{y}\|},
$$

and $\|\cdot\|$ is the Euclidian norm in the threedimensional space.

By applying the two trace operators (11), (12) to the equation (13), one can formulate a system of integral equations with the unknowns: $\gamma_{0}^{\Omega} u$ и $\gamma_{1}^{\Omega} u$. To formally define the mentioned system, the half-integer Sobolev spaces are introduced:

$$
\begin{gathered}
H^{1 / 2}(\Gamma)=\left\{g \mid g=\gamma_{0}^{\Omega} f, f \in H^{1}(\Omega)\right\} ; \\
H^{-1 / 2}(\Gamma)=\left\{g \mid g=\gamma_{1}^{\Omega} f, f \in H^{1}(\Omega)\right\},
\end{gathered}
$$

where $H^{1}(\Omega)$ is the Sobolev space of differentiable functions defined on $\Omega$. For the details related to the half-integer Sobolev spaces see [27]. Let us introduce as well the linear boundary integral operators $V_{k}, K_{k}, K_{k}^{a d d}$ and $D_{k}$ following [10]. The single layer operator $V_{k}$ is defined as follows:

$$
\begin{gathered}
\left(V_{k} f\right)(\mathbf{y})=\int_{\Gamma} G_{k}(\mathbf{y}, \mathbf{x}) f(\mathbf{x}) \mathrm{d} \Gamma_{\mathbf{x}}, \\
V_{k}: H^{-1 / 2}(\Gamma) \rightarrow H^{1 / 2}(\Gamma),
\end{gathered}
$$


the adjoint double layer operator $\left(K_{k}^{\prime} f\right)$ :

$$
\begin{gathered}
\left(K_{k}^{\prime} f\right)(\mathbf{y})=\int_{\Gamma} \gamma_{1, \mathbf{y}}^{\Omega} G_{k}(\mathbf{y}, \mathbf{x}) f(\mathbf{x}) \mathrm{d} \Gamma_{\mathbf{x}}, \\
K_{k}^{\prime}: H^{-1 / 2}(\Gamma) \rightarrow H^{-1 / 2}(\Gamma),
\end{gathered}
$$

the double layer operator $K_{k}$ :

$$
\begin{gathered}
\left(K_{k} f\right)(\mathbf{y})=\int_{\mathbf{x} \in \Gamma} \gamma_{1, \mathbf{x}}^{\Omega} G_{k}(\mathbf{y}, \mathbf{x}) f(\mathbf{x}) \mathrm{d} s_{\mathbf{x}}, \\
K_{k}: H^{1 / 2}(\Gamma) \rightarrow H^{1 / 2}(\Gamma),
\end{gathered}
$$

and the hypersingular operator $D_{k}$ :

$$
\begin{gathered}
\left(D_{k} f\right)(\mathbf{y})=\gamma_{1, \mathbf{y}} \int_{\mathbf{x} \in \Gamma} \gamma_{1, \mathbf{x}}^{\Omega} G_{k}(\mathbf{y}, \mathbf{x}) f(\mathbf{x}) \mathrm{d} s_{\mathbf{x}}, \\
D_{k}: H^{1 / 2}(\Gamma) \rightarrow H^{-1 / 2}(\Gamma) .
\end{gathered}
$$

Here's also the definition of the duality pairing between the half-integer Sobolev spaces $H^{1 / 2}$ and $H^{-1 / 2}$ :

$$
\langle u, w\rangle=\int_{\mathbf{x} \in \Gamma} u(\mathbf{x}) \bar{w}(\mathbf{x}) \mathrm{d} s_{\mathbf{x}}, u \in H^{1 / 2}, w \in H^{-1 / 2} .
$$

Using relations (15)-(18), the Galerkin representation of integral equation can be obtained in the following form [10]:

$$
\begin{array}{r}
\left\langle V_{k} \gamma_{1}^{\Omega} u, w\right\rangle=\left\langle\left(\frac{1}{2} I+K_{k}\right) \gamma_{0}^{\Omega} u, w\right\rangle, \forall w \in H^{-1 / 2}(\Gamma) ; \\
\left\langle D_{k} \gamma_{0}^{\Omega} u, v\right\rangle=\left\langle\left(\frac{1}{2} I-K_{k}^{\prime}\right) \gamma_{1}^{\Omega} u, v\right\rangle, \forall v \in H^{1 / 2}(\Gamma) .
\end{array}
$$

If there's only the Dirichlet data function defined on $\Gamma$ then through the substitution of the known data into (20) one derives the variational problem with the Neumann data as the only unknown. The variational problem (21) allows determining the Dirichlet data when the Neumann data is predefined. The later problem is solvable and has a unique solution only when the number $-k^{2}$ is not an eigenvalue of the Laplace operator [28]. When the two conditions are mixed on the border of $\Omega$ then a variational problem has to be solved. This problem can be formulated in terms of the Steklov - Poincare operator [10]:

$$
\left\langle S_{k} \gamma_{0}^{\Omega} u, v\right\rangle=\left\langle\gamma_{1}^{\Omega} u, v\right\rangle,
$$

where $S_{k}$ is defined as follows:

$$
S_{k}=D_{k}+\left(\frac{1}{2} I+K_{k}^{\prime}\right) V_{k}^{-1}\left(\frac{1}{2} I+K_{k}\right),
$$

and the test function $v$ is from the space of functions, that are equal to zero on $\Gamma_{1}$.
The discretization of (20) and (21) is possible via projecting the unknown data to the finite dimensional subspaces $U_{h}\left(\Gamma_{h}\right) \subset H^{1 / 2}\left(\Gamma_{h}\right)$ и $W_{h}\left(\Gamma_{h}\right) \subset H^{-1 / 2}\left(\Gamma_{h}\right)$, where $\Gamma_{h}$ may stand for a surface mesh which geometry approximates $\Gamma, h$ is a discretization parameter.

Let the dimension of $U_{h}\left(\Gamma_{h}\right)$ be equal to $N$ and the dimension $W_{h}\left(\Gamma_{h}\right)$ be equal to $M$ respectively. Let also $g_{p}, p=1, N$ be the basis functions in $U_{h}\left(\Gamma_{h}\right), w_{q}$, $q=1, M$ - the basis functions in $W_{h}\left(\Gamma_{h}\right)$. In order to construct the corresponding discreate system, one can approximate the Dirichlet and Neumann data using the linear combinations of the vectors belonging to the corresponding finite-dimensional subspaces:

$$
\begin{aligned}
& \gamma_{0}^{\Omega} u(\mathbf{x}) \approx \sum_{p=1}^{N} \alpha_{p} g_{p}(\mathbf{x}), g_{p} \in U_{h}(\Gamma) \\
& \gamma_{1}^{\Omega} u(\mathbf{x}) \approx \sum_{q=1}^{M} \beta_{q} w_{q}(\mathbf{x}), w_{q} \in W_{h}(\Gamma) .
\end{aligned}
$$

Substituting (24), (25) into (20), (21) one derives a SLAE:

$$
\left(\begin{array}{cc}
\mathbf{V} & -\mathbf{K} \\
\mathbf{K}^{\mathrm{T}} & \mathbf{D}
\end{array}\right)\left(\begin{array}{l}
\boldsymbol{\beta} \\
\boldsymbol{\alpha}
\end{array}\right)=\left(\begin{array}{l}
0 \\
\Phi
\end{array}\right),
$$

where $\alpha$ is a vector of coefficients $\alpha_{p}$ in decomposition (24); $\beta-$ vector of coefficients $\beta_{q}$ in (25). SLAE blocks in (26) can be expressed as follows:

$$
\begin{gathered}
\mathbf{V}_{i, j}=\left\langle V_{k} w_{i}, w_{j}\right\rangle, i, j=1, M ; \\
\mathbf{D}_{i, j}=\left\langle D_{k} g_{i}, g_{j}\right\rangle, i, j=1, N ; \\
\mathbf{K}_{i, j}=\left\langle\left(\frac{1}{2} I+K_{k}\right) g_{i}, w_{j}\right\rangle, i=1, N, j=1, M ; \\
\boldsymbol{\Phi}_{i}=\left\langle F_{N}, g_{i}\right\rangle, i=1, N .
\end{gathered}
$$

The indirect integration of the function $G_{k}$ stands in the formulae (27)-(29) because of the definitions (15)-(18). This is why the computation of (27)-(29) is not trivial. The traditional methods of numerical integration are inapplicable to the problem of computing the correct values because the fundamental solution $G_{k}(\mathbf{x}, \mathbf{y})$ is not continuous when the arguments $\mathbf{x}$ and $\mathbf{y}$ are equal. Different solutions to this problem are suggested in [29-35].

\section{FEM and BEM comparison conducted via model problems}

As a part of the computer program implementation of BEM, a mesh composed out of triangular 
elements was exploited to approximate the boundary of the computational domains specified for the model problems. The basis functions $g_{q}$ of $W_{h}\left(\Gamma_{h}\right)$ used to approximate the Neumann data were chosen to be piecewise constant functions equal to one only on their corresponding local supports that are triangles of the mesh $\Gamma_{h}$. The basis functions $w_{p}$ of $U_{h}\left(\Gamma_{h}\right)$ are piecewise linear functions. See more about the basis functions in [10]. For the finite element method program implementation, the quadratic basis was chosen. See more about quadratic basis implementation for the Helmholtz equation solved via FEM in [36].

To test the efficiency of the computation strategies, let us consider the model problems described below.

The first model problem geometry looks like this: in a closed domain of a cube with $20 \mathrm{~m}$ length of its edges, a ball of radius equal to $1.5 \mathrm{~m}$ and a central point coincident with the center of the cube is situated. The wave number $k$ in (8) is equal to $2 \mathrm{~m}^{-1}$, which corresponds to the case of the sound speed equal to $400 \mathrm{~m} / \mathrm{s}$ and the frequency equal to $127.32 \mathrm{~Hz}$. The boundary conditions for all the boundary parts can be expressed as follows:

$$
\left.u(\mathbf{x})\right|_{\mathbf{x} \in \Gamma}=\cos (k x),
$$

where $x$ is a coordinate of $\mathbf{x}$ along $O X$ axis. Every axis of the Cartesian coordinate system is parallel to one of the edges of the cube. It's clear that with conditions (31) the corresponding analytic solution of (8) takes a form

$$
u(\mathbf{x})=\cos (k x)
$$

everywhere in the computation domain.

The surface mesh (Fig. 1) used for BEM computations consists of 3284 elements. The number of nodes is equal to 1646 . The volume mesh (Fig. 2) used for the FEM computations is composed out of 17133 elements. The corresponding number of nodes is 26007 .

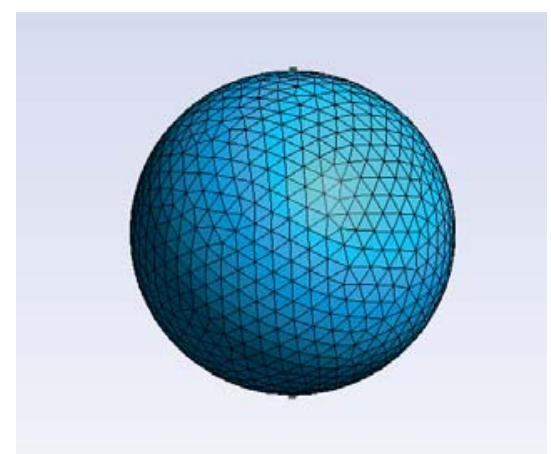

Fig. 1. An example of the surface mesh of the sphere used for the BEM computations

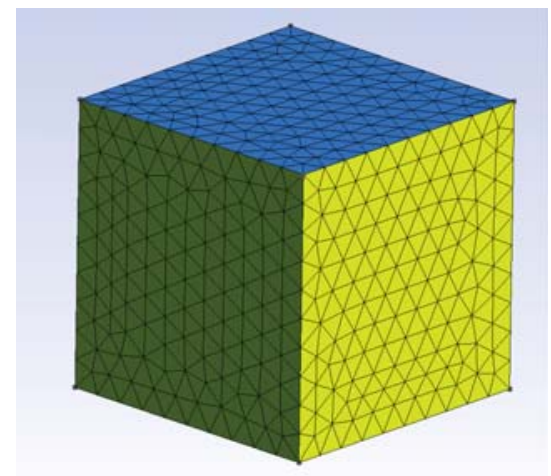

Fig. 2. An example of the cube mesh used for the FEM computations

Figure 3 illustrates the curves of relative discrepancies of the solutions resulted from the implementation of numerical approaches in relation with the analytic solution. The values are given at the points situated along the $O X$ axis. The value of relative discrepancy is equal to:

$$
\frac{u(\mathbf{x})-u^{*}(\mathbf{x})}{\max \left|u^{*}\right|}
$$

As one can see, the solution resulted from BEM turns out to be more accurate than the one obtained with FEM.

Let's solve the problem for which the analytic solution is known. An incident wave in a medium is represented as follows:

$$
\Psi_{i n c}=e^{i(-\mathbf{k} \cdot \mathbf{x})},
$$

where $i$ is an imaginary one; $\mathbf{k}$ is the direction of the incident wave; $\mathbf{x}$ - radius vector characterizing

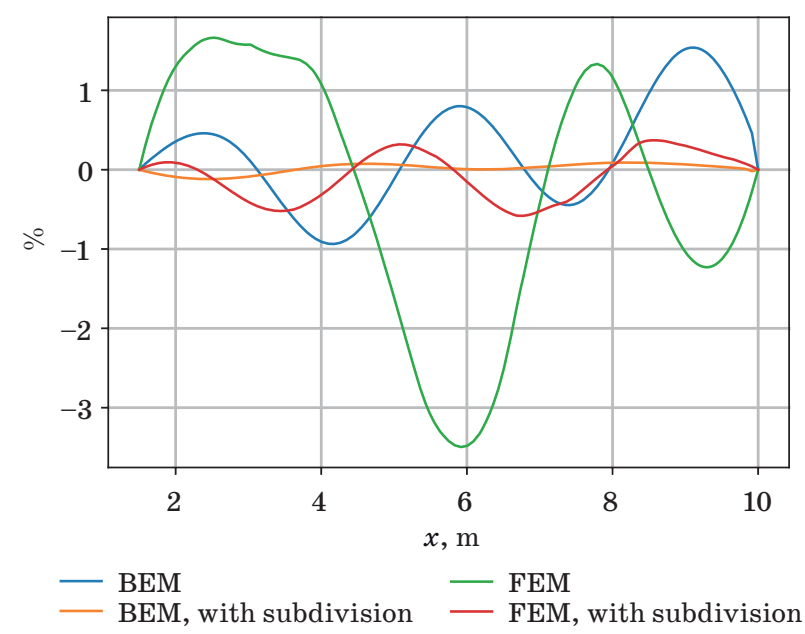

- Fig. 3. The numerical errors' curves produced for the plane wave solution compared with the numerical methods' results working with the original mesh and its subdivision 
the position in space. The spheric boundary of the ball is the source of the scattered wave $\Psi_{s c}$. The sum of $\Psi_{s c}$ and $\Psi_{i n c}$ is denoted as $\Psi$. Let the Dirichlet condition be imposed on the sphere:

$$
\left.\Psi\right|_{\Gamma_{s}}=\Psi_{i n c}+\left.\Psi_{s c}\right|_{\Gamma_{s}}=0
$$

or

$$
\left.\Psi_{s c}\right|_{\Gamma_{s}}=-\left.\Psi_{i n c}\right|_{\Gamma_{s}}=0
$$

where $\Gamma_{s}$ is the spheric boundary of the ball.

The scattered wave should then take a form [37]:

$$
\begin{gathered}
\Psi_{s c}(\mathbf{x})=\sum_{m=0}^{\infty}(2 m+1) i^{-m} P_{m}(\cos (\theta)) \times \\
\times\left(j_{m}(k\|\mathbf{x}\|)-\frac{j_{m}(k a)}{h_{m}(k a)} h_{m}(k\|\mathbf{x}\|)\right)-\Psi_{i n c}(\mathbf{x}),
\end{gathered}
$$

where $P_{m}$ - the Legendre polynomial of order $m$; $\theta$ is the angle between $\mathbf{x}$ and $\mathbf{k} ; j_{m}$ - the spherical Bessel function of order $m$; $k$ - the modulus of $\mathbf{k}$ or its length; $a$ - the sphere radius; $h_{m}-$ the spherical Hankel function of order $m$.

To compare (34) with the numerical solution of the Helmholtz equation, one has to take as an approximation of $\Psi_{s c}(\mathbf{x})$ a sum composed out of a finite number of terms in (34):

$$
\begin{gathered}
\Psi_{K}(\mathbf{x})=\sum_{m=0}^{K}(2 m+1) i^{-m} P_{m}(\cos (\theta)) \times \\
\times\left(j_{m}(k r)-\frac{j_{m}(k a)}{h_{m}(k a)} h_{m}(k r)\right)-e^{-\mathbf{x} \cdot \mathbf{k}} .
\end{gathered}
$$

To make such comparison possible, the value of $K$ was chosen to be equal to 20 , which provides six digits of the approximation accuracy.

Let the value of $k$ be equal to $0.1 \mathrm{~m}^{-1}$. To be able to solve this problem, FEM requires the area of significant volume to be set up. In our framework, it's a cube with the edge length equal to $20 \mathrm{~m}$. That's why the FEM geometry of the computation domain is left unchanged in comparison with the previous problem description. The cube boundary also requires the Dirichlet condition to be homogeneous. The BEM variation of this problems is solved in the open region.

Figure 4 illustrates that the numerical discrepancy grows for FEM when the point argument approaches the border of the computation domain that is far away from the sphere. The set of points used for the comparison (see Figs. 3 and 4) is the same.

Figure 5 demonstrates the curves of relative discrepancy only for the case of BEM computations.

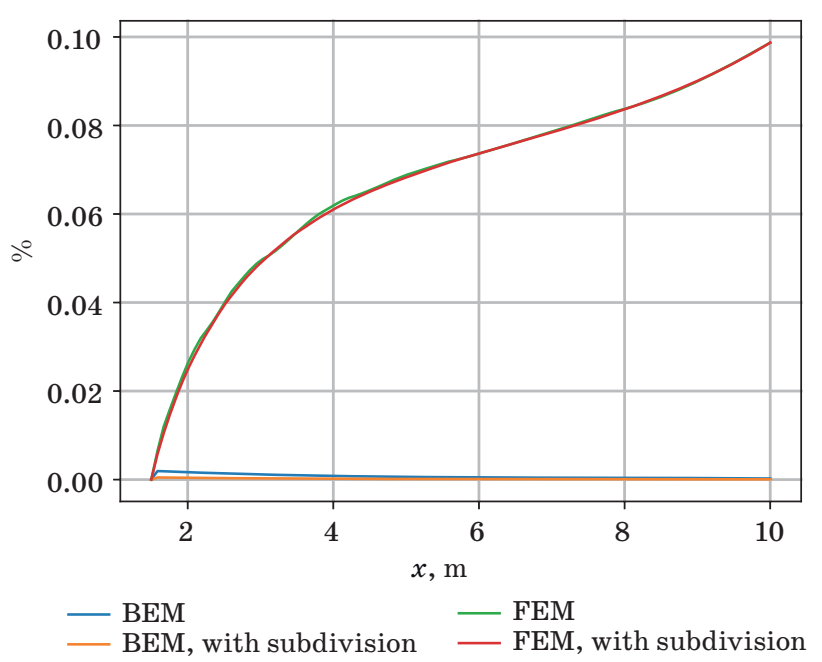

Fig. 4. The scattered wave numerical error curves

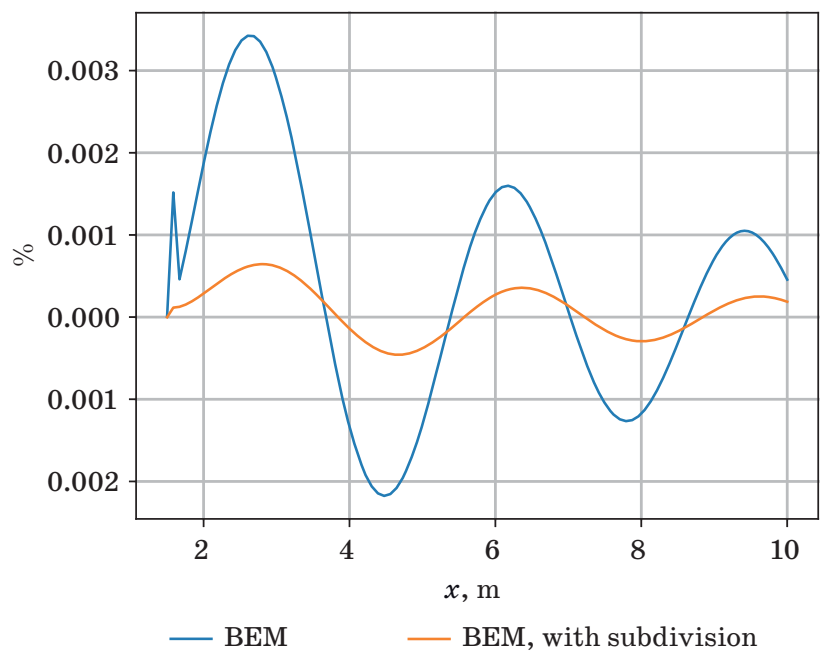

Fig. 5. The comparison for the scattered wave with the wave number equal to $2 \mathrm{~m}^{-1}$

FEM requires a significant number of volume elements in case of the border distant from the sphere when $k=2 \mathrm{~m}^{-1}$. That's why we were unable to obtain adequate FEM results.

\section{Conclusion}

The program implementation of the BEM allowing the Helmholtz equation to be solved in bounded and unbounded regions has been developed. The validity of this approach has been tested for internal and external Dirichlet problems. The comparison with analytics demonstrates effectiveness of BEM relatively to FEM because the latter requires a fine mesh to be used in the computation domains of significant volume. 


\section{References}

1. Chen L. H., Schweikert D. G. Sound radiation from an arbitrary body. The Journal of the Acoustical society of America, 1963, no. 10, pp. 1626-1632. doi:10.1121/ 1.1918770

2. Banaugh R. P., Goldsmith W. Diffraction of steady acoustic waves by surfaces of arbitrary shape. The Journal of the Acoustical Society of America, 1963, no. 10, pp. 1590-1601. doi:10.1121/1.1918764

3. Chertock G. Sound radiation from vibrating surfaces. The Journal of the Acoustical Society of America, 1964, no. 7, pp. 1305-1313. doi:10.1121/1.2142487

4. Greenspan D., Werner P. A numerical method for the exterior Dirichlet problem for the reduced wave equation. Archive for Rational Mechanics and Analysis, 1966, no. 4, pp. 288-316. doi:10.1007/BF00281165

5. Copley L. G. Integral equation method for radiation from vibrating bodies. The Journal of the Acoustical Society of America, 1967, no. 4A, pp. 807-816. doi:10. 1121/1.1910410

6. Hsiao G. C., Wendland W. L. Super-approximation for boundary integral methods. Proc. of the 4th IMACS Conf. on Computer Mem. for Part. Diff. Equ., 1981, pp. 200-205.

7. Wendland W. L. On asymptotic error estimates for combined BEM and FEM. In: Stein E., Wendland W. (Eds.) Finite element and boundary element techniques from mathematical and engineering point of view. Springer-Verlag, New York, 1988. Pp. 273-333. doi:10.1002/zamm.19900701019

8. Demkowicz L., Karafiat A., Oden J. T. Solution of elastic scattering problems in linear acoustics using hp boundary element method. Computer Methods in Applied Mechanics and Engineering, 1992, no. 1-3, pp. 251-282. doi:10.1016/0045-7825(92)90025-F

9. Geng P., Oden J. T., Demkowicz L. Numerical solution and a posteriori error estimation of exterior acoustics problems by a boundary element method at high wave numbers. The Journal of the Acoustical Society of America, 1996, no. 1, pp. 335-345. doi:10.1121/1.415883

10. Rjasa now S., Steinbach O. The fast solution of boundary integral equations. Springer Science \& Business Media, 2007. $291 \mathrm{p}$.

11. Coox L., Atak O., Vandepitte D., Desmet W. An isogeometric indirect boundary element method for solving acoustic problems in open-boundary domains. Computer Methods in Applied Mechanics and Engineering, 2017, pp. 186-208. doi:10.1016/j.cma.2016.05.039

12. Simpson R. N., Scott M. A., Taus M., Thomas D. C., Lian H. Acoustic isogeometric boundary element analysis. Computer Methods in Applied Mechanics and Engineering, 2014, pp. 265-290. doi:10.1016/j. cma.2013.10.026

13. Harbrecht H., Schneiderd R. Biorthogonal wavelet bases for the boundary element method. Mathematische Nachrichten, 2004, vol. 261, no. 1, pp. 167-188. doi:10.1002/mana.200310171
14. Wang G. A hybrid wavelet expansion and boundary element analysis of electromagnetic scattering from conducting objects. IEEE Transactions on Antennas and Propagation, 1995, vol. 43, no. 2, pp. 170-178. doi:10.1109/8.366379

15. Koro K., Abe K. Non-orthogonal spline wavelets for boundary element analysis. Engineering Analysis with Boundary Elements, 2001, vol. 25, no. 3, pp. 149-164. doi:10.1016/S0955-7997(01)00036-4

16. Harbrecht H., Utzinger M. On adaptive wavelet boundary element methods. Journal of Computational Mathematics, 2018, vol. 36, no. 1, pp. 90-109. doi:10.4208/jcm.1610-m2016-0496

17. Kurz S., Rain O., Rjasanow S. The adaptive cross-approximation technique for the 3D boundary-element method. IEEE Transactions on Magnetics, 2002, no. 2, pp. 421-424. doi:10.1109/20.996112

18. Brancati A., Aliabadi M. H., Benedetti I. Hierarchical adaptive cross approximation GMRES technique for solution of acoustic problems using the boundary element method. Computer Modeling in Engineering and Sciences (CMES), 2009, vol. 38, no. 2, pp. 149172. doi:10.1109/20.996112

19. Smajic J., Andjelic Z., Bebendorf M. Fast BEM for eddy-current problems using $\mathrm{H}$-matrices and adaptive cross approximation. IEEE Transactions on Magnetics, 2007, vol. 43, no. 4, pp. 1269-1272. doi:10.1109/ TMAG.2006.890971

20. Coifman R., Rokhlin V., Wandzura S. The fast multipole method for electromagnetic scattering calculations. Proceedings of IEEE Antennas and Propagation Society International Symposium, 1993, pp. 4851. doi:10.1109/APS.1993.385405

21. Shen L., Liu Y. J. An adaptive fast multipole boundary element method for three-dimensional acoustic wave problems based on the Burton-Miller formulation. Computational Mechanics, 2007, no. 3, pp. 461472. doi:10.1007/s00466-006-0121-2

22. Gumerov N. A., Duraiswami R. Fast multipole methods for the Helmholtz equation in three dimensions. Elsevier, 2005. $551 \mathrm{p}$.

23. Gumerov N. A., Duraiswami R. Fast, exact, and stable computation of multipole translation and rotation coefficients for the 3-d Helmholtz equation. 2001, Available at: https://drum.lib.umd.edu/bitstream/handle/1903/1141/ CS-TR-4264.pdf?sequence $=1$ (accessed 8 August 2020).

24. Sivak S. A., Stupakov I. M., Kondratieva N. S. A combined vector method of finite and boundary elements for simulating electromagnetic filed propagation considering an eddy current model. Science Bulletin of the Novosibirsk State Technical University, 2018, no. 4(73), pp. 7990 (In Russian). doi:10.17212/1814-1196-2018-4-79-90

25. Stupakov I. M., Royak M. E., Bubley P. A. Using fast multipole method for magnetic field calculation in complex system of current coils. 2018 XIV International Scientific-Technical Conference on Actual Problems of Electronics Instrument Engineering (APEIE), 2018, pp. 307-310. doi:10.1109/APEIE.2018.8545530 
26. Stupakov I., Royak M., Kondratyeva N. Coupled finite and boundary element method for solving magnetic hysteresis problems. Transactions on Engineering Sciences, 2019, pp. 125-135. doi:10.2495/be420111

27. Adams R. A., Fournier J. J. F. Sobolev spaces. Academic Press, New York, 2003. 317 p.

28. Stolper M. Computing and compression of the boundary element matrices for the Helmholtz equation. Journal of Numerical Mathematics, 2004, no. 1, pp. 55-75. doi:10.1515/1569395041172935

29. Keller P. A method for indefinite integration of oscillatory and singular functions. Numerical Algorithms, 2007, no. 3, pp. 219-251. doi:10.1007/s11075-007-9134-y

30. Assari P., Adibi H., Dehghan M. The numerical solution of weakly singular integral equations based on the meshless product integration (MPI) method with error analysis. Applied Numerical Mathematics, 2014, pp. 76-93. doi:10.1016/j.apnum.2014.02.013

31. Nair N. V., Pray A. J., Villa-Giron J., Shanker B., Wilton D. R. A singularity cancellation technique for weakly singular integrals on higher order surface descriptions. IEEE Transactions on Antennas and Propagation, 2013, no. 4, pp. 2347-2352. doi:10.1109/TAP.2013. 2238880

32. Cancela A. C. Transformation methods for the integration of singular and near-singular functions in XFEM. Doctoral Dissertation, Tesis Doctoral, Universidad Na- cional de Educaci n a Distancia (UNED) Faculty of Science Department of Statistics, Operations Research and Numerical Analysis, 2017. Available at: http://62.204. 194.43/fez/eserv/tesisuned:Ciencias-Acano/CANO_CANCELA_Alfredo_Tesis.pdf (accessed 5 January 2021).

33. Vasconcelos A. C. A., Cavalcante I., Labaki J. On the accuracy of adaptive quadratures in the numerical integration of singular Green's functions for layered media. Proceedings of the Iberian Latin American Congress on Computational Methods in Engineering, 2017. doi:10.20906/cps/cilamce2017-0063

34. Järvenp S., Taskinen M., Yl-Oijala P. Singularity extraction technique for integral equation methods with higher order basis functions on plane triangles and tetrahedra. International Journal for Numerical Methods in Engineering, 2003, no. 8, pp. 1149-1165. doi:10.1002/nme.810

35. Huang S., Liu Y. J. A new fast direct solver for the boundary element method. Computational Mechanics, 2017, no. 3, pp. 379-392. doi:10.1007/s00466-017-1407-2

36. Solovejchik J. G., Rojak M. E., Persova M. G. Metod konechnyh jelementov dlja reshenija skaljarnyh $i$ vektornyh zadach [The finite element method for solving the scalar and vector problems]. Novosibirsk, NSTU Publ., 2007. 896 p. (In Russian).

37. Lependin L. F. Akustika [Acoustics]. Moscow, Vysshaja shkola Publ., 1978. 448 p. (In Russian).

УДК 519.633.6, 519.642.2

doi:10.31799/1684-8853-2021-2-13-19

\section{Использование метода граничных элементов при решении уравнения Гельмгольца для задачи акустики}

С. А. Сивак ${ }^{\mathrm{a}}$, аспирант, orcid.org/0000-0003-4740-2210, siwakserg@yandex.ru

М. Э. Рояк ${ }^{\mathrm{a}}$, доктор техн. наук, профессор, orcid.org/0000-0001-8304-7784

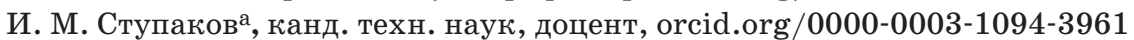

Е. С. Вознюк ${ }^{\mathrm{a}}$, аспирант, orcid.org/0000-0001-7362-6002

А. С. Алексашина ${ }^{\mathrm{a}}$ магистрант, orcid.org/0000-0003-1871-5517

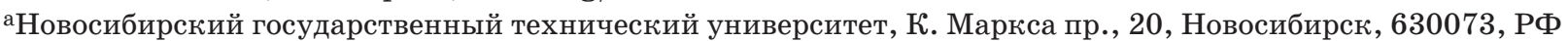

Введение: решение уравнения Гельмгольца представляет практическую значимость для отраслей, в которых требуется моделирование волновых процессов. Использование численных методов позволяет повысить эффективность проводимых расчетов. Методы: для численного решения уравнения Гельмгольца можно использовать метод граничных элементов. Для его применения необходимо построить только поверхностную сетку границы трехмерной области, в которой решается задача. Данная особенность позволяет производить расчеты в том числе и во внешней области по отношению к некоторой ограниченной замкнутой подобласти трехмерного евклидова пространства, что также дает возможность обходиться без дополнительных геометрических построений, необходимых для учета бесконечно удаленной границы. Однако расчет методом граничных элементов возможно проводить только для однородной области либо для множества смежных однородных областей. Результаты: разработана реализация метода граничных элементов для решения уравнения Гельмгольца применительно к задаче акустики в рамках программного комплекса Quasar. Проанализировано отклонение результатов, полученных методом граничных элементов для внутренней и внешней краевых задач, от приближенной аналитики. Приводятся также результаты, полученные при решении модельных задач методом конечных элементов, для сравнения двух различных подходов. Практическая значимость: данный метод позволяет решать уравнение Гельмгольца в неограниченной области, что является большим преимуществом по сравнению с численными методами, требующими объемной дискретизации расчетной области, и, в частности, с методом конечных элементов. Обсуждение: в дальнейшем планируется осуществить комбинирование методов граничных и конечных элементов для расчетов в неограниченной подобласти с постоянными параметрами среды и в расчетных подобластях, чья среда является существенно неоднородной.

Ключевые слова - метод граничных элементов, метод конечных элементов, уравнение Гельмгольца, акустика.

Для цитирования: Sivak S. A., Royak M. E., Stupakov I. M., Voznuk E. S., Aleksashin A. S. The implementation of the boundary element method to the Helmholtz equation of acoustics. Информационно-управляющие системы, 2021, № 2, с. 13-19. doi:10.31799/16848853-2021-2-13-19

For citation: Sivak S. A., Royak M. E., Stupakov I. M., Voznuk E. S., Aleksashin A. S. The implementation of the boundary element method to the Helmholtz equation of acoustics. Informatsionno-upravliaiushchie sistemy [Information and Control Systems], 2021, no. 2, pp. 13-19. doi:10.31799/1684-8853-2021-2-13-19 